\title{
ДИСКУССИИ
}

\section{Учет отраслевых факторов при анализе инвестиционного поведения компаний (на примере нефтегазовой отрасли)}

\author{
Родионов И.И., ${ }^{13}$ Протасов В.С. ${ }^{14}$
}

Важность инвестиций в современной экономике обуславливает необходимость увеличения точности исследований по ним, что может быть достигнуто благодаря учету отраслевой специфики. В статье обоснована значимость отраслевых факторов для инвестиционного поведения компаний, найдень и систематизированы факторы для нефтегазовой отрасли, приведены рекомендации по эмпирическим исследованиям.

JEL: G31

Ключевые слова: инвестиции, инвестиционное поведение, нефть, газ

\section{Введение}

Одним из ключевых параметров, который определяет экономическое развитие во всем мире, являются инвестиции. Как следствие крайней значимости инвестиций и инвестиционного поведения компаний, которое является объектом исследования в настоящей работе, их изучением занимались сотни авторов (см., в частности, табл. 1). Тем не менее до сих пор не существует единой устоявшейся теории, которая полностью объясняла бы инвестиционное поведение компаний и динамику инвестиций в целом, а результаты различных исследователей зачастую кардинально отличаются друг от друга. Одной из возможных причин относительно низкого уровня объясняющей силы существующих моделей может являться недостаточный учет специфических отраслевых факторов, влияющих на инвестиции. Для проверки этой гипотезы следует подробно, на качественном уровне изучить специфику основных отраслей экономики с точки зрения инвестиционного поведения. В настоящем исследовании для этого была выбрана нефтегазовая отрасль. Выбор предмета настоящего исследования - факторов инвестиционного поведения нефтяных и газовых компаний - обусловлен высокой значимостью этой отрасли для России, так как она обеспечивает около 20\% ВВП и 60\% экспорта страны [ИЭС, 2008]. Цель исследования обосновать важность учета специфических отраслевых факторов при анализе инвестиционного поведения компаний в целом и нефтегазовой отрасли в частности. Для достижения цели необходимо: выявить факторы, особенно, специфические, влияющие на инвестиции компаний нефтегазовой отрасли, проанализировать воздействие найденных факторов на уровень и структуру инвестиций, обобщить и систематизировать данные по влиянию факторов на инвестиционное поведение.

\section{Классификация факторов инвестиционного поведения}

Под инвестиционным поведением в настоящей работе будет пониматься совокупность решений компании о размере, структуре и временном распределении капитальных вложений. Основу современного анализа инвестиционного поведения компаний заложили еще Модильяни и Миллер [Modigliani, Miller, 1958], которые сводили решения фирм об

\footnotetext{
${ }^{13}$ Профессор кафедры экономики и финансов фирмы ГУ ВШЭ, д-р эконом. наук.

14 Аспирант экономического факультета ГУ ВШЭ, старший эксперт Института энергетики и финансов.
} 
инвестициях к стремлению собственников к максимизации своего благосостояния. С тех пор спектр рассматриваемых факторов значительно расширился, что обуславливает необходимость их систематизации. Проанализировав проделанные ранее исследования инвестиционного поведения (табл. 1) и выделив рассматривавшиеся в них факторы, можно их систематизировать, разделив в первую очередь на внутренние и внешние, а последние на общеэкономические и отраслевые (рис. 1). Другой вариант во многом аналогичной классификации приведен в работе Тепловой и Крыловой [Теплова, Крылова, 2007].

Таблица 1

Факторы инвестиционного поведения в рассмотренных исследованиях с примерами

\begin{tabular}{|c|c|c|c|}
\hline Фактор & Исследования & Фактор & Исследования \\
\hline $\begin{array}{l}\text { Экономические } \\
\text { циклы }\end{array}$ & $\begin{array}{l}\text { Long, Plosser, 1987; Blackley, } \\
\text { 2000; Philippon, } 2006\end{array}$ & Долговой рынок & $\begin{array}{l}\text { Gilchrist, Himmelberg, } \\
\text { 1995; Brown, Petersen, } \\
2008\end{array}$ \\
\hline $\begin{array}{l}\text { Технологические } \\
\text { шоки }\end{array}$ & Cooper, Haltiwanger, 1990 & Дивидендная политика & Moyen, 2004 \\
\hline Инфляция & $\begin{array}{l}\text { Goel, Ram, 2001; Pindyck, } \\
1991\end{array}$ & $\begin{array}{l}\text { Операционные } \\
\text { издержки }\end{array}$ & $\begin{array}{l}\text { Hartman, 1972; } \\
\text { Kalckreuth, 2000 }\end{array}$ \\
\hline Налоги & $\begin{array}{l}\text { MacKie-Mason, 1990; Rodrik, } \\
\text { 1991; Metcalf, Hassett, 1994; } \\
\text { Pennings, 2000; Hamaaki, } 2008\end{array}$ & \multirow[b]{2}{*}{$\begin{array}{l}\text { Внутренняя } \\
\text { ликвидность }\end{array}$} & \multirow{2}{*}{$\begin{array}{l}\text { Fazzari et. al., 1988, 2000; } \\
\text { Zingales, Kaplan, 1997; } \\
\text { Moyen, 2004; Povel, } \\
\text { Raith, 2001; Ascioglu, } \\
\text { 2008; Smith, Valderrama, } \\
\text { 2009 }\end{array}$} \\
\hline Обменные курсы & Fan, Zhu, 2010 & & \\
\hline $\begin{array}{l}\text { Государственная } \\
\text { политика (кроме } \\
\text { налоговой политики) } \\
\text { и инвестиционный } \\
\text { климат }\end{array}$ & $\begin{array}{l}\text { Wilson, 1987; Fan, Zhu, 2010; } \\
\text { Fattouh, Darbouche, } 2010\end{array}$ & $\begin{array}{l}\text { Структура } \\
\text { собственности, тип } \\
\text { акционера }\end{array}$ & $\begin{array}{l}\text { Lazareva et.al., 2008; } \\
\text { Удальцов, 2007; } \\
\text { Malmendier, 2001, } 2005\end{array}$ \\
\hline Ценовые шоки & $\begin{array}{l}\text { Fuss, Vermeulen, 2004; Abel, } \\
\text { 1983, 1997; Hartman, 1972; } \\
\text { Dixit, 1991 }\end{array}$ & $\begin{array}{l}\text { Эффективность } \\
\text { менеджмента, проблема } \\
\text { принципал-агент }\end{array}$ & $\begin{array}{l}\text { Удальцов, 2007; } \\
\text { Philippon, 2006; } \\
\text { Malmedier, 2001, } 2005\end{array}$ \\
\hline Шоки спроса & $\begin{array}{l}\text { Fuss, Vermeulen, 2004; } \\
\text { Sterken, 2002; Caballero, } 1991\end{array}$ & Доходность бизнеса & $\begin{array}{l}\text { Теплова, Крылова, 2007; } \\
\text { Osmundsen et al., } 2006\end{array}$ \\
\hline Фондовый рынок & $\begin{array}{l}\text { Shiantarelli, 1989; Schaller, } \\
\text { 1990; Bond et. al, 1992; } \\
\text { Gilchrist, 2005; Osmundsen et } \\
\text { al., } 2006\end{array}$ & $\begin{array}{l}\text { Инвестиционные } \\
\text { издержки }\end{array}$ & $\begin{array}{l}\text { Gould, 1968; Hartman, } \\
\text { 1972; Abel, Eberly, 1997; } \\
\text { Valles, } 1997\end{array}$ \\
\hline
\end{tabular}




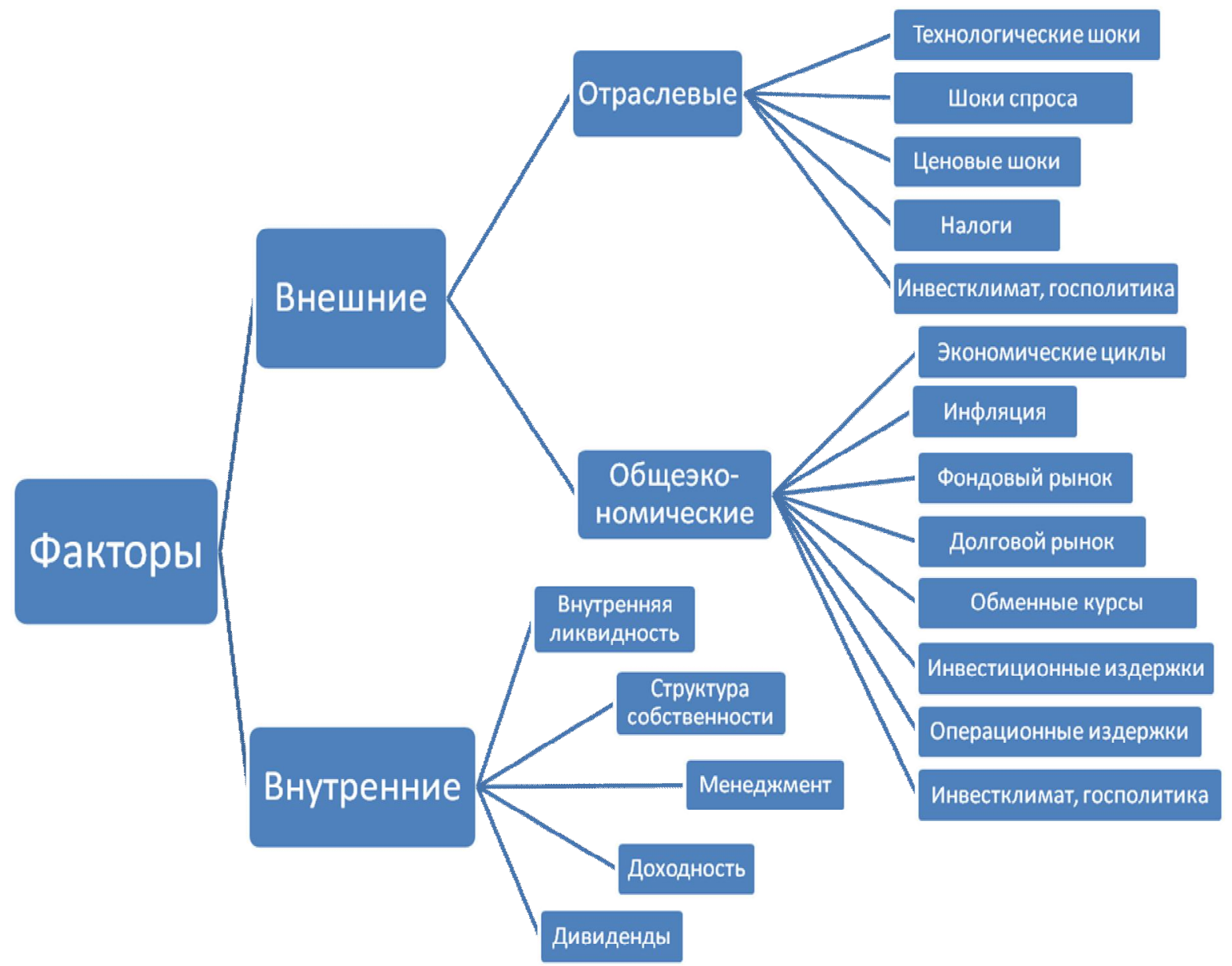

Рисунок 1. Классификация факторов инвестиционного поведения

Относительно перечисленных в таблице 1 работ можно сделать три обобщения:

1. По каждому из указанных факторов существует хотя бы одна работа, доказывающая его значимость.

2. Ни одна из работ не пытается учесть влияния всех перечисленных факторов.

3. Работы различных исследователей зачастую опровергают друг друга. В частности, наиболее примечательным примером является так называемый спор Фаззари Зингалеса [см., например: Fazzari et al., 1988, 2000; Zingales, Kaplan, 1997].

Таким образом, в рассматриваемой области исследований отсутствует единая система, описывающая влияние факторов на инвестиционное поведение компаний, наблюдается противоречивость результатов, что может быть обусловлено недостаточным учетом отраслевой специфики.

\section{Исследования отраслевых особенностей инвестиционного поведения}

Согласно исследованию Лонга и Плоссера [Long, Plosser, 1987]. отраслевые, а не общеэкономические шоки обуславливают около $80 \%$ колебаний основных показателей (инвестиций, выпуска, занятости) в отраслях. Данный вывод подтверждается в работ Блэкли [Blackley, 2000], где делается вывод о том, что отраслевые шоки объясняют 72\% инвестиций в среднем по 9 секторам экономики США. С другой стороны, другие авторы [Сoоper, Haltiwanger, Power, 1999] для американских промышленных предприятий сделали вывод о большей значимости общеэкономических шоков, хотя авторы также отмечают, что отсутствие учета секторальных шоков приведет к значительным ошибкам в результатах. Отметим, что в последней работе рассматриваемые компании не распределены по отраслям, поэтому ее нельзя сравнить по отраслевой структуре с работой Блэкли, что могло бы 
объяснить расхождение. Шапиро и Глоберман [Shapiro, Globerman, 2003] также отмечают важность отраслевого анализа при изучении инвестиций, обосновывая это тем, что воздействие государства на различные отрасли значительно различается

Следует отметить не только важность учета отраслевых факторов, но и относительно большую их важность для анализа некоторых секторов по сравнению с другими. В частности, Блэкли [Blackley, 2000] показал, что для добывающей промышленности США (в которую входит и нефтегазовая отрасль) отраслевыми шоками объясняются 89\% изменения инвестиций (в то же время в производстве потребительских товаров уже общие шоки обуславливают 70\% изменения инвестиций).

В исследованиях по инвестиционному поведению нефтяных и газовых компаний система рассматриваемых факторов, как правило, значительно отличается от прочих работ. Фан и Жу [Fan, Zhu, 2010] отмечают, что инвестиции в нефтяной отрасли являются сложным процессом с рядом специфических характеристик, таких как большой размер капитальных вложений, длительный период строительства и высокая неопределенность. При этом авторы выделяют три основных фактора, которые оказывают влияние на инвестиционную политику: нефтяные цены (наиболее важный фактор), инвестиционный климат и обменные курсы. В работе Мон и Мисанд [Mohn, Misund, 2009] показано, что включение в регрессионный анализ по инвестициям нефтегазовых компаний специфического отраслевого фактора (волатильности цен на нефть) и волатильности фондового рынка привело к тому, что показатель Q Тобина (стандартный показатель, используемый в теории корпоративных финансов) стал незначим. В результате авторы делают вывод о том, что Q Тобина является плохим показателем для изучения инвестиций нефтяных и газовых компаний. Осмундсен [Osmundsen et al., 2006] утверждает, что финансовые рынки оказывают значительное влияние на нефтяные и газовые компании и, возможно, накладывают ограничения на их инвестиции, так как стимулируют улучшение краткосрочных показателей, таких как RoACE (доход на средний используемый капитал). Таким образом, финансовые рынки создают инвестиционные ограничения для нефтегазовых компаний, причем, исходя из логики исследования, не только по абсолютному размеру инвестиций, но и по их временной структуре (относительно больший размер инвестиций с коротким сроком окупаемости, что позволяет увеличивать RoACE).

Отметим высокое значение политической составляющей при принятии инвестиционных решений в нефтегазовой отрасли, что проявляется, в частности, в нефтеполитических циклах [Wilson, 1987]. В условиях высоких цен на нефть страны экспортеры углеводородов получают большие финансовые ресурсы, что увеличивает их влиятельность на переговорах и ведет к усилению государственного контроля над отраслью (и наоборот). Тем самым нефтеполитические циклы оказывают влияние на институциональные инвестиционные ограничения и барьеры. Современные подтверждения существования нефтеполитического цикла могут быть найдены в исследованиях отечественных авторов [Гурвич, Вакуленко, Кривенко, 2009].

Выявление общих для нефтегазовой отрасли закономерностей влияния факторов на инвестиционное поведение может быть затруднено существованием своей специфики по странам и типам компаний. В работе Фаттух и Дарбуш [Fattouh, Darbouche, 2010] описывается специфика инвестиционного поведения нефтегазовых компаний для Алжира, Ливии, Египта и Судана, а также показывается, что нефтеполитический цикл определяется индивидуально по странам в зависимости от возможностей национальных нефтяных компаний и накопленных финансовых резервов правительства. Согласно Локателли [Locatelli, 2006], важной характеристикой российских нефтяных компаний в 1990-2000-х годах является низкая доля инвестиций в геологоразведку, что во многом обусловлено стратегией «вывода денег». Во второй половине 2000-х годов поведение нефтегазовых компаний России стало приближаться к практике европейских и американских компаний, хотя этому значительно помешало «дело Юкоса». Хартли и Медлок [Hartley, Medlock, 2007] доказали, что долгосрочное инвестиционное поведение национальных нефтяных компаний 
(ННК) показывает наличие систематических отличий от поведения международных нефтяных компаний.

\section{Факторы инвестиционного поведения нефтегазовых компаний}

Для анализа инвестиционного поведения нефтегазовых компаний можно предложить следующую систему факторов (при их описании подробнее остановимся на более специфичных):

1. Основной драйвер инвестиций - цена нефти.

2. Финансовые возможности:

- рентабельность бизнеса;

- внутренняя ликвидность компании (операционный доход, финансовые резервы);

- возможности привлечения внешнего финансирования (заемные средства, внутренний рынок, поддержка государства);

- стоимость инвестиционных проектов.

3. Инвестиционные ограничения:

- институциональные барьеры;

- $\quad$ природные ограничения;

- $\quad$ размер запасов углеводородов в компании.

4. Инвестиционные перспективы:

- стратегия компании;

- среднесрочные и долгосрочные прогнозы спроса на нефть и природный газ, их продукты;

- $\quad$ риски инвестирования.

5. Инвестиционные обязательства.

Цена нефти - универсальный фактор для нефтегазовой отрасли, который оказывает влияние на все прочие группы факторов: определяет уровень рентабельности, размер денежных потоков, возможности привлечения внешнего финансирования (поставки нефти как залог, цена акций), налоги (привязка налогов к цене нефти), стоимость инвестиционных проектов и инвестиционные обязательства (через спрос на инвестиционные товары и услуги), институциональные барьеры (нефтеполитический цикл), размер запасов, прогнозы спроса, риски компании (волатильность цен на нефть). Следует отметить, что для большинства других отраслей наблюдается прямая зависимость цены нефти (через цены на нефтепродукты, природный газ, электроэнергию) и издержек компании и, соответственно, отрицательная зависимость с финансовыми возможностями и инвестициями [см., в частности: Glass, Cahn, 1987]). При этом экспортная и оптовая цена на природный газ в мире сильно (80-90\%) коррелирована с ценами на нефть на мировом рынке [Секретариат Энергетической Хартии, 2007; Stern, 2007]. Для нефтепереработки, нефте- и газохимии зависимость от цены нефти обратная, поэтому они часто используются нефтегазовыми компаниями как возможность диверсификации своего инвестиционного портфеля для снижения рисков [Helfat, 1989].

В нефтегазовой отрасли особенно велика роль государства. Поддержка государства с точки зрения финансовых возможностей может выражаться следующими способами: предоставление государственными структурами субсидий, льготных кредитов, правительственных гарантий по кредитам, передача государственных активов в собственность компании, предоставление монопольного положения (например, монополия «Газпрома» на экспорт природного газа из России).

Инвестиционные издержки влияют на размер инвестиций и их межвременную структуру [Abel, Eberly, 1997]. При этом динамика стоимости инвестиционных товаров и услуг в отрасли может значительно отличаться от общеэкономической инфляции или стоимости инвестиционных товаров. В частности, в 2006 году инфляция в мире составила $3,5 \%$, а стоимость инвестиционных проектов в секторе разведки и разработки увеличилась на 
31,2\% [IMF, 2010; Протасов, 2009].

По аналогии с финансовыми ограничениями (financial constraints) компаний можно ввести термин инвестиционные ограничения. Их существование обуславливается природными, технологическими и институциональными причинами, а также ограниченностью запасов углеводородов, принадлежащих компании (рис. 2). Природные причины: геологические - истощение запасов сырья, нехватка разведанных месторождений; и геоклиматические - расположение месторождений на столь значительном расстоянии от существующей инфраструктуры и потребителей или в столь тяжелых природных условиях, что их разработка будет нерентабельной. Отсутствие технологий для разработки углеводородов, находящихся в определенной геологической формации (нефтяные и газовые сланцы, метан угольных пластов, газ плотных коллекторов) или химически связанном виде (газогидраты) обуславливает невозможность их разработки в определенный момент времени и тем самым создает ограничения по инвестированию в них. В зависимости от наличия запасов (вероятных и возможных) могут планироваться инвестиции в геологоразведку в среднесрочной и в разработку в долгосрочной перспективе. Ряд месторождений в мире запрещено разрабатывать, так как они отнесены к стратегическим запасам или для охраны окружающей среды. Кроме инвестиционного климата также следует выделить наличие институциональных барьеров для определенной группы инвесторов, то есть ограничений или запретов для иностранных инвесторов на вложения в нефтегазовые активы определенной страны (характерно для стран ОПЕК, России, Мексики). Инвестиционные ограничения приводят к снижению уровня инвестиций в отрасли (либо инвестиций определенного типа компаний) относительно оптимального для них уровня. При снятии части институциональных ограничений (например, в условиях экономического кризиса) может возникнуть эффект отложенных инвестиций.

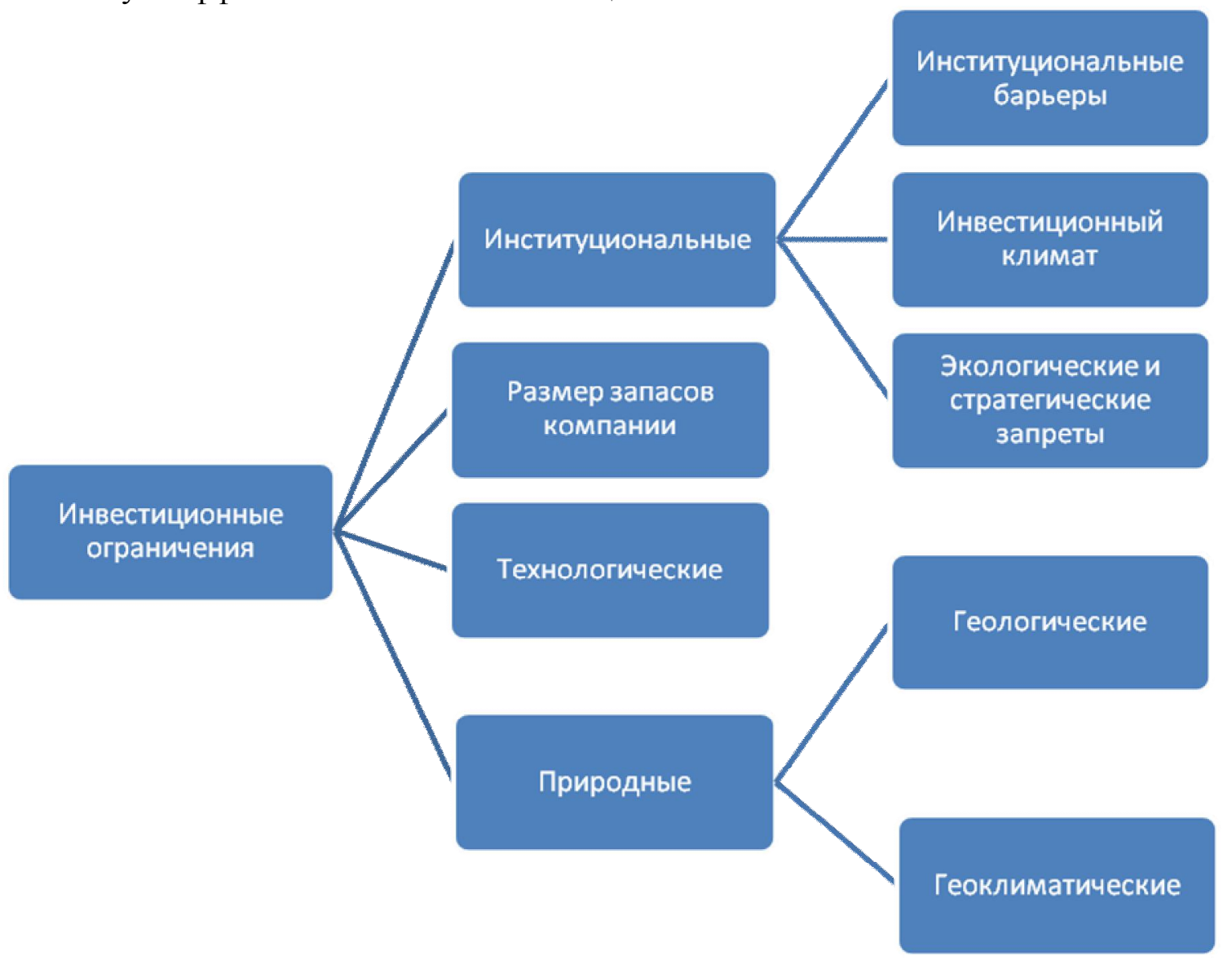

Рисунок 2. Инвестиционные ограничения нефтегазовых компаний 
Стратегия компании во многом определяет, в какой степени финансовые возможности компании будут использованы для инвестирования (и какова будет структура инвестиций), какой уровень рентабельности будет считаться достаточным и как будут интерпретированы перспективы роста спроса. При этом в отраслях можно выделять типовые стратегии инвестиционного поведения. В частности, для нефтегазовой отрасли типовые стратегии можно определять для мэйджоров, национальных нефтегазовых компаний и прочих частных компаний (возможно и более подробное деление) [Протасов, 2009]. Прогнозы спроса на нефть и газ являются важнейшим параметром, определяющим цены на текущий момент и в долгосрочной перспективе.

Важным свойством инвестирования в нефтегазовую отрасль является ее инерционность, которая обусловлена:

- д длительным сроком реализации проектов при высокой специфичности инвестиций;

- лицензионными требованиями;

- для поддержания (и тем более увеличения) уровня добычи углеводородов в компании, стране и мире необходимо постоянное инвестирование в новые проекты из-за истощения старых месторождений;

- геологическими и технологическими особенностями реализации проектов по добыче углеводородов.

Как следствие, у каждой компании на определенный момент времени существуют инвестиционные обязательства, то есть минимальный уровень инвестиций, которые компания должна обеспечить для продолжения своего нормального функционирования или во избежание потерь. Они могут достигать до 75\% объема капитальных вложений компании за год [ВР, 2009]. Инерционность инвестиций обуславливает высокую значимость показателя инвестиций предыдущих периодов при регрессионном анализе, а также то, что явления немонотонности (lumpiness) инвестиций нехарактерны для компаний отрасли. Фактор инвестиционных обязательств может быть применен в качестве эмпирического обоснования

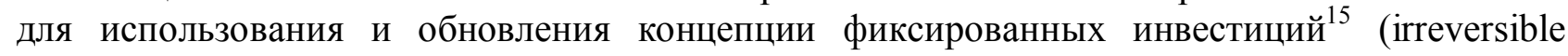
investments) [Bernanke, 1983; Dixit, 1991; Goel, Ram, 2001; Pindyck, 1991]. Следует заметить, что существует проблема причинно-следственной связи между инвестиционными обязательствами и инвестиционной программой. Действительно, в зависимости от среднесрочных планов компании по ее инвестиционной программе могут заключаться сервисные контракты, арендоваться оборудование, что напрямую влияет на размер инвестиционных обязательства. Тем не менее в условную точку принятия решения о размере инвестиций на следующий год размер существующих инвестиционных обязательств является заданным, поэтому он принимается во внимание в инвестиционном поведении.

Важно отметить, что факторы инвестиционных обязательств и ограничений в академической литературе ранее не рассматривались. Вероятно, это во многом связано с тем, что они являются достаточно специфическими факторами, характерными для небольшого числа отраслей, инвестиционное поведение в которых исследовано недостаточно. Кроме того, это может быть обусловлено сложностью получения количественных данных по указанным факторам.

Таким образом, капитальные вложения компаний нефтегазовой отрасли ограничены инвестиционными нормами и обязательствами - сверху и снизу соответственно. Многие компании могут существовать внутри ограниченной области, не достигая в обычных обстоятельствах этих границ, так как их финансовые возможности достаточны для вложений сверх обязательств (а инвестиционные перспективы стимулируют к этому), но не позволяют достичь границы инвестиционных ограничений. Однако изменение внешних условий способно привести к проявлению указанных факторов. В частности, экономический кризис может вынудить уменьшить вложения до уровня обязательств.

\footnotetext{
15 Данный термин также можно перевести как необратимые или безвозвратные инвестиции, но в этом случае их можно спутать с понятием «sunk cost», которое несет совершенно другую смысловую нагрузку.
} 


\section{Заключение}

Проведенный анализ, систематизация и классификация исследований инвестиционного поведения позволяют сделать вывод об отсутствии единой системы, описывающей влияние факторов на него, противоречивости результатов существующих работ. Выдвинута гипотеза о том, что одна из причин этого - отсутствие учета или недостаточный учет отраслевой принадлежности и специфики исследуемых компаний.

В соответствии с результатами ряда исследований (хотя существуют и противоположные мнения), отраслевые факторы оказывают существенно большее влияние на инвестиционное поведение компаний, чем общеэкономические. При этом в добывающих отраслях они могут объяснять до 90\% динамики инвестиций.

Исследователи выделяют ряд специфических отраслевых факторов (цена нефти, нефтеполитический цикл, инвестиционный климат и государственная политика в отрасли), а также особенностей нефтегазовой отрасли (большой размер капитальных вложений, длительный период строительства, высокая неопределенность). Но при этом не создано единых подходов к анализу и общего механизма инвестиционного поведения нефтегазовых компаний. Высокая специфичность инвестиционного поведения компаний нефтегазовой отрасли обуславливает необходимость рассмотреть ее отдельно

Многие исследования описывают группы компаний нефтегазовой отрасли, обладающих своим типом поведения. Группы могут классифицироваться по признаку наличия государства как контролирующего акционера, принадлежности к определенной стране или группе стран. Специфичность их инвестиционных стратегий, которую можно выделить и подробно изучить при рассмотрении отдельно компаний нефтегазовой отрасли, будет крайне затруднительно учесть при рассмотрении компаний всех отраслей, обладающих собственными типами компаний и имеющих свою инвестиционную стратегию.

Автором предложена новая классификация факторов инвестиционного поведения, учитывающая специфику нефтегазовой отрасли. Все факторы разделены на пять групп: цена нефти, финансовые возможности, инвестиционные ограничения, обязательства и перспективы. При этом введены два новых типа факторов инвестиционного поведения, ранее не рассматривавшиеся исследователями: инвестиционные ограничения и инвестиционные обязательства, характерные для нефтяных и газовых компаний. Новые факторы с точки зрения теории инвестиций могут увеличить объясняющую силу моделей, помочь в интерпретации коэффициентов, дать толчок развитию концепции фиксированных инвестиций. Теоретически обоснована высокая значимость показателя инвестиций предыдущих периодов при эконометрическом анализе капитальных вложений нефтегазовых компаний. Предложена типология компаний нефтегазовой отрасли, которая может быть включена в качестве объясняющей переменной в количественное исследование для отражения фактора инвестиционной стратегии. Существование инвестиционных ограничений также может поставить под вопрос правильность интерпретации результатов при использовании концепции финансовых ограничений.

В целом можно сделать вывод о необходимости включения в эмпирические исследования инвестиционного поведения фактора отрасли, проведения отдельных исследований по всем основным секторам экономики с учетом их специфики. Данный вывод ставит под сомнение достоверность результатов ранее проведенных исследований, которые не учитывали отраслевую специфику, особенно если в выборку были включены нефтегазовые компании и компании ряда других отраслей, имеющих свои особенности инвестиционного поведения. Учет отраслевого фактора позволит увеличить объясняющую силу моделей. При рассмотрении инвестиционного поведения нефтегазовой отрасли в качестве факторов должны быть включены цена нефти (либо показатели на ее основе, например, волатильность цен на нефть), инвестиции предыдущих периодов. При эмпирическом анализе можно рассмотреть более сложные функциональные формы 
зависимости, учитывающие существование инвестиционных ограничений и обязательств.

\section{Список литературы}

1. Гурвич Е., Вакуленко Е., Кривенко П. Циклические свойства бюджетной политики в нефтедобывающих странах // Вопросы экономики. 2009. № 9. С. 51-70.

2. Концепция Энергетической стратегии России на период до 2030 г. (проект) // Прил. к научн., обществ.-дел. журналу «Энергетическая политика». М.: ГУ ИЭС, 2007. - 116 с.

3. Протасов В. Анализ инвестиционного поведения нефтегазовых компаний в условиях экономического кризиса // Сборник докладов Первого Российского экономического конгресса. М.: Институт Экономики РАН, 2009.

4. Секретариат Энергетической Хартии. Цена энергии. Брюссель: Секретариат Энергетической Хартии, 2007.

5. Теплова Т.В., Крылова М.С. Эмпирическое исследование факторов, определяющих инвестиционную активность российских компаний // Корпоративные финансы. 2007. № 1.

6. Удальцов В.Е. Влияние доли топ-менеджмента в собственном капитале и других нефундаментальных факторов на инвестиционную активность российских компаний // Корпоративные финансы. 2007. № 1.

7. Abel, A.B. (1983), Optimal investment under uncertainty, American Economic Review, 73 (1983) 228-233.

8. Abel, A.B., Eberly, J.C. (1997), An exact solution for the investment and value of a firm facing uncertainty, adjustment costs, and irreversibility, Journal of Economic Dynamics and Control, 21 (1997) 831-852.

9. Ascioglu, A., Hegde, Sh. P., McDermott, J. B. (2008), Information asymmetry and investment-cash flow sensitivity, Journal of Banking \& Finance, 32 (2008) 1036-1048.

10. Bernanke, B. (1983), Irreversibility, uncertainty and cyclical investment, Quarterly Journal of Economics, 98 (1983) 85-106.

11. Blackley, P. R. (2000), Sources of sectoral fluctuations in business fixed investment, Journal of Economics and Business, 52( 6) (2000) 473-484.

12. Bond, S., Devereux, M., Schiantarelli, F., Blundell, R. (1992), Investment and Tobin's Q: Evidence from company panel data, Journal of Econometrics, 51 (1992).

13. BP. Annual Review (2009).

URL: http://www.bp.com/assets/bp_internet/globalbp/globalbp_uk_english/set_branch/STAGING /common_assets/downloads/pdf/BP_Annual_Review_2009.pdf.

14. Brown, J., Petersen, B. (2009), Why has the investment-cash flow sensitivity declined so sharply? Rising R\&D and equity market developments, Journal of Banking \& Finance, 33 (2009) 971-984.

15. Caballero, R.J. (1991), On the sign of the investment - uncertainty relationship, American Economic Review, 81 (1991) 279-288.

16. Cooper, R., Haltiwanger, J. (1990), Inventories and the propagation of sectoral shocks, American Economic Review, 80 (1) (1990) 170-190.

17. Dixit, A. (1991), Irreversible investment with price ceilings, Journal of Political Economy, 99 (1991) 541-557.

18. Fazzari, S.R., Hubbard, G., and Petersen, B. (2000), Investment - cash Flow Sensitivities are Useful: A comment on Kaplan and Zingales, Quarterly Journal of Economics, 115 (2000) 695-705.

19. Fazzari, S., Hubbard, R.G., and Petersen, B. (1988), Financing Constraints and Corporate Investment, Brookings Papers on Economic Activity, (1988) 141-195.

20. Fan, Y., Zhu, L. (2010), A real options based model and its application to China's overseas oil investment decisions, Energy Economics, 32(3) (2010). 
21. Fattouh, B., Darbouche, H. (2010), North African oil and foreign investment in changing market conditions, Energy Policy, 38(2) (2010) 1119-1129.

22. Fuss, C., Vermeulen, P. (2004), Firms' investment decisions in response to demand and price uncertainty, NBB Working Paper, 45 (2004).

23. Gilchrist, S., Himmelberg, C. (1995), Evidence on the role of cash flow for investment, Journal of Monetary Economics, 36 (1995).

24. Gilchrist, S., Himmelberg, Ch.P., Huberman, G. (2005), Do stock price bubbles influence corporate investment? , Journal of Monetary Economics, 52 (2005) 805-827.

25. Goel, R., Ram, R. (2001), Irreversibility of R\&D investment and the adverse effect of uncertainty: Evidence from the OECD countries, Economics Letters, 71(2) (2001) 287-291.

26. Gould, J. P. (1968), Adjustment costs in the theory of investment of the firm, Review of Economic Studies, 35 (1968) 47-56.

27. Glass, V., Cahn, E. S. (1987), Energy prices and investment over the business cycle, Energy Economics, 9(4) (1987) 257-264.

28. Hamaaki, J. (2008), Investment responses to Japanese tax reforms: A cross-industry comparison, Japan and the World Economy, 20(4) (2008) 542-562.

29. Hartley, P., Medlock, K. (2008), A model of the operation and development of a National Oil Company, Energy Economics, 30(5) (2008) 2459-2485.

30. Hartman, R. (1972), The effect of price and cost uncertainty on investment, Journal of Economic Theory, 5 (1972) 258-266.

31. Helfat, C. (1989), Investment in offshore oil by diversified petroleum companies, Journal of industrial economics, 38 (1989).

32. International Monetary Fund, World Economic Outlook Database (2010). URL: http://www.imf.org/external/pubs/ft/weo/2010/02/weodata/index.aspx.

33. Kalckreuth, U. (2000), Exploring the Role of Uncertainty for Corporate Investment Decisions in Germany, Discussion paper 5/00 (2000).

34. Kaplan, S.N., Zingales, L. (1997), Do investment-cash flow sensitivities provide useful measures of financing constraints, Quarterly Journal of Economics, 112 (1997) 169-215.

35. Lazareva, O., Rachinsky, A., Stepanov, S. (2008), Corporate Governance, Ownership Structures and Investment in Transition Economies: the Case of Russia, Ukraine and Kyrgyzstan, Working Paper № 119, CEFIR / NES Working paper series (2008).

36. Locatelli, C. (2006), The Russian oil industry between public and private governance: obstacles to international oil companies' investment strategies, Energy Policy, 34(9) (2006) $1075-1085$.

37. Long, J., Plosser, C. (1987), Sectoral vs. aggregate shocks in the business cycle, American Economic Review, 77(2) (1987) 333-336.

38. MacKie-Mason, J.K. (1990), Some nonlinear tax effects on asset values and investment decisions under uncertainty, Journal of Public Economics, 42 (1990) 301-328.

39. Malmendier, U., Geoffrey, T. (2005), Does Overconfidence Affect Corporate Investment? CEO Overconfidence Measures Revisited, European Financial Management, 11(5) (2005).

40. Metcalf, G., Hassett, K. (1994), Investment with uncertain tax policy. Working paper no. 4780, NBER, Cambridge, MA.

41. Modigliani, F., Miller, M. (1958), The Cost of Capital, Corporation Finance and the Theory of Investment, American Economic Review, 48(3) (1958) 261-297.

42. Mohn, K., Misund, B. (2009), Investment and uncertainty in the international oil and gas industry, Energy Economics, 31(2) (2009) 240-248.

43. Moyen, N. (2004), Investment-cash flow sensitivities: constrained versus unconstrained firms, The Journal of finance, 59 (2004).

44. Osmundsen, P., Asche, F., Misund, B., Mohn, K. (2006), Valuation of oil companies, Energy Journal, 27 (2006) 49-64.

45. Pennings, E. (2000), Taxes and stimuli of investment under uncertainty, European Economic Review, 44(2) (2000) 383-391. 
46. Philippon, T. (2006), Corporate governance over the business cycle, Journal of Economic Dynamics and Control, 30(11) (2006) 2117-2141.

47. Povel, P., Raith, M. (2002), Optimal Investment Under Financial Constraints: the Roles of Internal Funds and Asymmetric Information, (2002).

48. Pindyck, R.S. (1991), Irreversibility, Uncertainty, and Investment, Journal of Economic Literature, 29(3) (1991) 1110-1148.

49. Rodrik, D. (1991), Policy uncertainty and private investment in developing countries, Journal of Development Economics, 36 (1991) 229-242.

50. Schaller, H. A. (1990), Re-examination of the Q Theory of Investment Using U.S. Firm Data, Journal of applied econometrics, 5(4) (1990).

51. Shapiro, D., and Globerman, S. (2003), Foreign investment policies and capital flows in Canada: a sectoral analysis, Journal of Business Research, 56(10) (2003) 779-790.

52. Shiantarelli, F. (1989), Devereux. Investment, financial factors and cash flow: evidence from UK panel data, NBER Working paper, 3116 (1989).

53. Smith, K. A., Valderrama, D. (2009), The composition of capital inflows when emerging market firms face financing constraints, Journal of Development Economics, 89(2) (2009) 223-234.

54. Sterken, E., Lensink, R., Bo, H. (2002), Investment, cash flow and uncertainty: Evidence for Netherlands, Preliminary version, Department of Economics University of Groningen The Netherlands, (2002).

55. Stern, J. (2007), Is There A Rationale for the Continuing Link to Oil Product Prices in Continental European Long-Term Gas Contracts?, OIES papers, 19 (2007).

56. Vallès, J. (1987), Aggregate investment in a business cycle model with adjustment costs, Journal of Economic Dynamics and Control, 21(7) (1997) 1181-1198.

57. Wilson III, E.J. (1987), World politics and international energy markets, International Organization, 41(1) (1987) 125-149. 